\title{
A comparison of monocular and 'stereoscopic' photographs of the optic disc in the identification of glaucomatous visual field defects
}

\author{
N. K. SHARMA AND R. A. HITCHINGS \\ From the Glaucoma Unit, Moorfields Eye Hospital, High Holborn, London WC1V 7AN
}

SUMMARY From a comparison of the results obtained by examining 'monocular' and 'stereoscopic' photographs of the optic disc it was concluded that each afforded similar levels of accuracy in identifying glaucomatous visual field defects.

To screen for glaucoma requires the ability to identify the changes at the optic disc and/or the visual field that suggest this disease, as well as the identification of elevated intraocular pressure. The identification of changes occurring at the optic disc has the advantage over visual field screening in that it is both rapid and independent of the patient's response. Visual field screening, by contrast, depends on the patient's response and may give rise to an unacceptably high number of false positives. ${ }^{1}$ An overlay grid superimposed on 'stereoscopic' optic disc photographs facilitates identification of the thin neuroretinal rim seen in patients with glaucomatous visual field defects. ${ }^{2}$ Because of the simplicity of this method of examination it can be used by nonmedically qualified individuals and thus would be suitable for the purpose of glaucoma screening. If 'stereoscopic' photographs of the optic disc were available they would form a useful adjunct to tonometry in glaucoma screening. However, because it is simpler to photograph the optic disc without trying for a 'stereoscopic' effect, it seemed worthwhile to try to identify those changes suggestive of glaucoma that are visible on monocular photographs of the optic disc and to compare the results with examination of stereoscopic pairs of optic disc photographs by means of an overlay grid.

\section{Material and methods}

Eighty-five photographs of the optic disc were used in this study. The photographs in each pair were obtained with a slight change in the alignment of the

Correspondence to Mr R. A. Hitchings, FRCS. camera body in relation to the eye so that a 'stereo' effect could be obtained when viewing the pair simultaneously. These photographs had been used in an earlier study, ${ }^{2}$ and the information on the presence or absence of visual field defects obtained on 3-isoptre Goldmann perimetry within 4 months of the photographs was available. The photographs were taken of patients attending the Glaucoma Unit at Moorfields Hospital, High Holborn, all of whom had initially presented with raised intraocular pressure.

The photographs were checked for visual field defects by one of us (N.S.) in 2 separate ways. Firstly, the paired photographs were looked at in conjunction with an overlay grid according to a method previously described $^{2}$; all eyes with a neuroretinal rim thickness of less than one division were identified and assumed to have visual field loss. Secondly, one photograph of each pair was looked at without the overlay grid, and on this occasion use was made of additional visual clues: 'angulation' of retinal vessels, extension of central cup pallor, ${ }^{3}$ and 'laminar dots' ${ }^{4}$ as well as visible thinning and undercutting of the neuroretinal rim (Fig. 1). For the purposes of this paper 'angulation' of a vessel on the surface of the optic disc was considered to be present if the vessel failed to travel on a straight line or smooth continuous curve over the surface of the optic disc. It was appreciated that the vessel could change direction in both the horizontal and as well as the anterioposterior plane. In these 'mono' photographs, however, the end result of these 2 possible changes in direction could be seen as a loss of the normal smooth course of a retinal blood vessel and as such would be recorded as 'angulation' (Fig. 1). The presence or absence of a visual field defect was not known to the observer at 


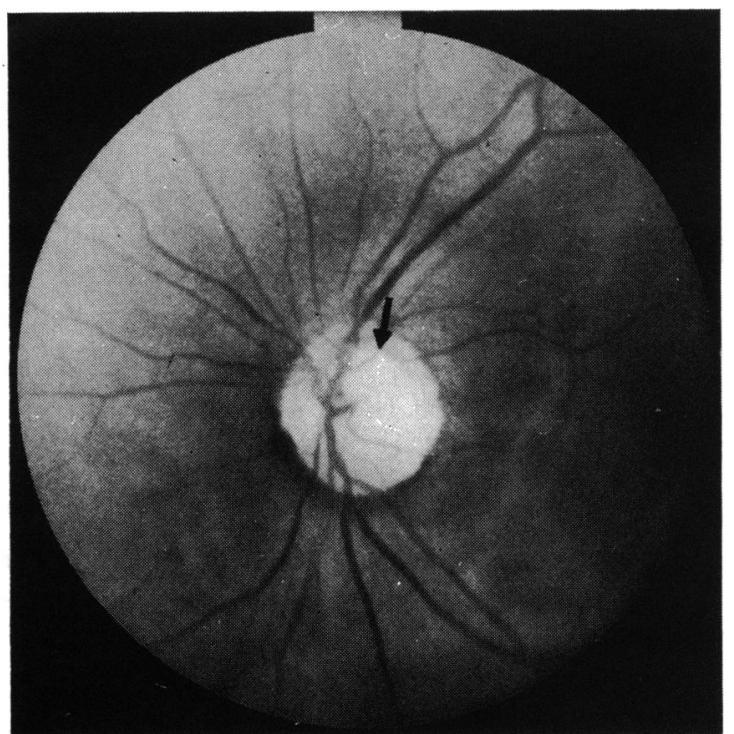

Fig. 1 Optic disc photograph showing angulation of blood vessels on the surface of optic nerve (arrow), pallor, and laminar dots.

the time of examining the optic discs. An interval of 2 weeks elapsed between the first and second sets of observations. When both sets of observations were finished, the presence or absence of a visual field defect for each optic disc was noted, and the results of the 2 series were compared.

\section{Results}

Eighty-five photographs of the optic disc were examined. The C/D ratios of this sample have been set out in Fig. 2, showing the number of eyes with

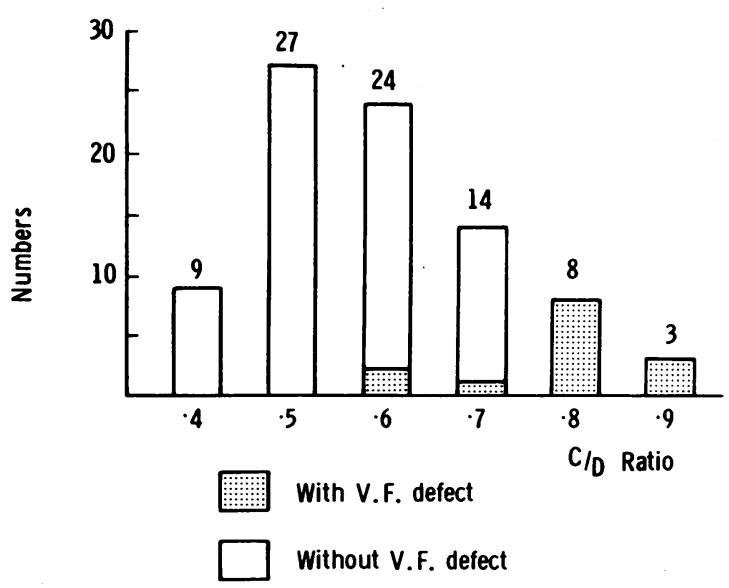

Fig. 2 Distribution of $C / D$ ratio in 85 eyes.

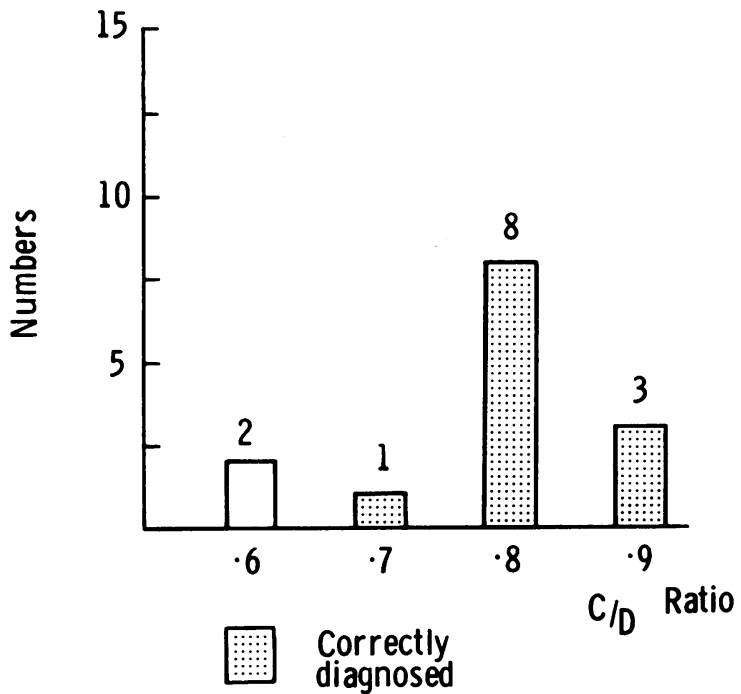

False VE

Fig. 3 Identification of optic discs with visual field defects.

each C/D ratio as well as the number with a visual field defect (14 in all).

Correct identification of those eyes with a visual field defect was made in 12 of 14 cases for both the 'monocular' photographs and the 'stereoscopic' disc pairs. The same 2 eyes were not recognised as having a field defect by either method of examination (Fig. 3).

Sixty-seven of the 71 eyes without a visual field defect were correctly predicted on examination of the stereo pairs. However, only 62 of them were correctly identified on examining the monocular photographs (Table 1).

\section{Discussion}

Examination of 'stereo' pairs combined with an overlay grid allowed correct identification of those glaucoma visual field defects noted on 3-isoptre perimetry. The improvement in the sensitivity and specificity in this series compared with that obtained

Table 1 Comparison of examining monocular and 'stereoscopic' photographs with false negative and false positive identification of visual field defects

\begin{tabular}{lcc}
\hline & Stereo & Mono \\
\hline Number & 85 & 85 \\
False negative & $2 / 14$ & $2 / 14$ \\
Sensitivity & $86 \%$ & $86 \%$ \\
False positive & $4 / 71$ & $9 / 71$ \\
Specificity & $95 \%$ & $87 \%$ \\
\hline
\end{tabular}




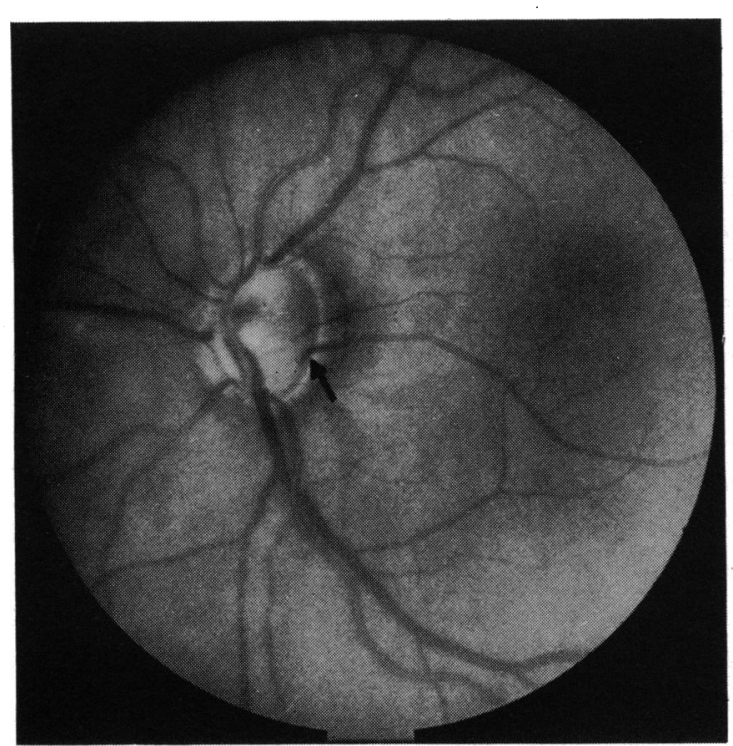

Fig. 4 Cilioretinal artery (arrow), incorrectly identified as angulation in an eye with a full visual field.

in an earlier report ${ }^{2}$ may reflect the subconscious use by a skilled observer of additional signs visible at the optic disc to identify a visual field defect. Two false negatives were seen in optic discs having a sloping cup. This created difficulty in identifying the precise location of the neuroretinal rim.

Examination of the monocular photographs

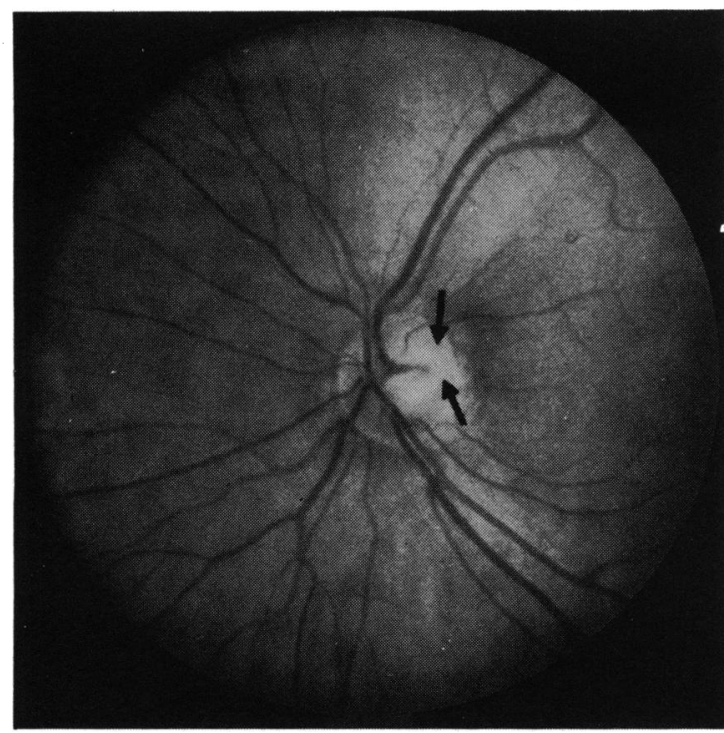

Fig. 5 Temporal extension of pallor (arrows) in an eye with a full visual field.

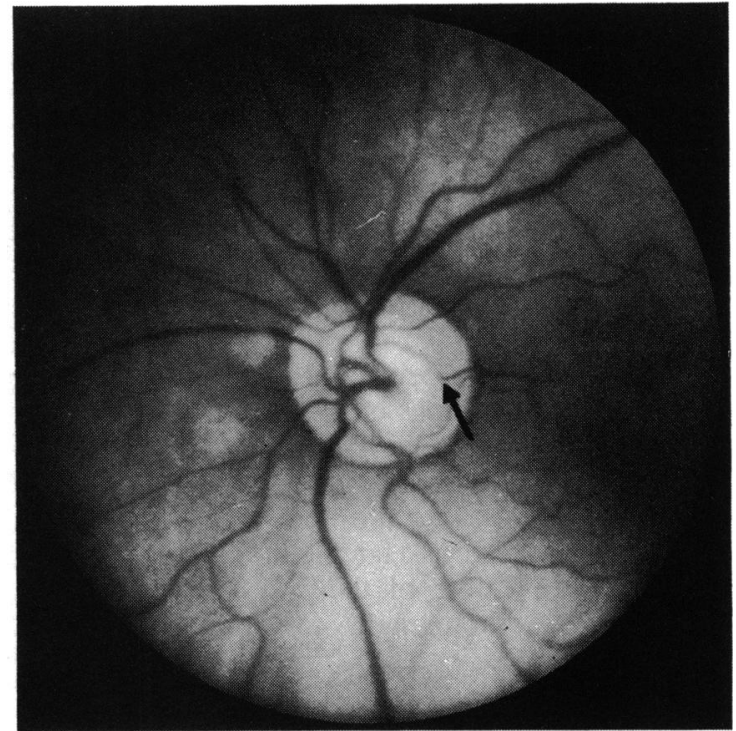

Fig. 6 Baring of circumlinear vessel and angulation (arrow) in an eye with a full visual field.

without the overlay grid meant that visual clues other than measured rim thickness had to be relied upon. These proved sufficient to identify correctly the same number of eyes with a known visual field defect. Reasons for the false positives were then identified. They include the presence of a cilioretinal artery misinterpreted as angulation (Fig. 4), temporal extension of the central cup pallor (Fig. 5), angulation of blood vessels (Fig. 6), and undercutting of the rim.

Frequently more than one of these signs were present at the optic disc, so that for the 8 eyes incorrectly stated to have a visual field defect the relative frequency of these signs could be identified (Table 2).

On reviewing the slides that had given rise to false positive identification of a visual field defect the authors considered the possibility that one or more of these eyes could have had an early visual field defect not identified by 3 -isoptre perimetry. In a study comparing static profile and 3-isoptre perimetry using a Goldmann bowl perimeter on patients with low tension and chronic simple glaucoma it was found

Table 2 Causes of false positive identification of visual field defects

\begin{tabular}{lll}
\hline Cilioretinal artery & 1 eye & Fig. 4 \\
Temporal extension of central cup pallor & 5 eyes & Fig. 5 \\
Angulation of optic disc vessels & 6 eyes & Fig. 6 \\
Poor focusing & 3 eyes & \\
\hline
\end{tabular}


that 3-isoptre perimetry (by the same method of examination as used for the patients in this study) missed 'absolute' (1000 asb) visual field defects in 14 of the 60 eyes examined. ${ }^{5} \mathrm{~A}$ review of the optic disc photographs of these patients (Hitchings, Anderton, ${ }^{6}$ in press) showed that a more accurate idea of the presence or absence of the visual field defects noted on static perimetry could be obtained from the appearance of the optic disc, noting the change outlined above. It seems possible, therefore, that a number of the false positives identified by examining monocular photographs of the optic disc did in fact have early visual field defects not identified by 3-isoptre perimetry on a Goldmann perimeter. Even if this is not the case, the results obtained from examining the high-risk sample in this study suggest that, by taking note of the appropriate clues on mononuclear photographs of the optic disc, glaucomatous visual field defects may be correctly identified and few will be missed.

\section{References}

1 Hitchings RA. Glaucoma Screening. Res Clin Forums 1981; 3: 109-14.

2 Hitchings RA, Anderton SA, Brown DB. Glaucoma screening using an optic disc grid. Br J Ophthalmol in press.

3 Shiose Y. 'Quantitative disc pattern' or a new parameter for glaucoma screening. Glaucoma 1979; 1: 41-9.

4 Read RM, Spaeth GL. The practical clinical appraisal of the optic disc in glaucoma: the natural history of cup progression and some specific disc-field correlations. Trans Am Acad Ophthalmol Otolaryngol 1974: 255-73.

5 Anderton SA, Hitchings RA. Comparison of visual fields of patients with low tension glaucoma and patients with chronic simple glaucoma. Presented at proceedings of International Perimetric Society, California 1982. In press.

6 Hitchings RA, Anderton SA. Identification of glaucomatous visual field defects from examination of monocular photographs of the optic disc. Br J Ophthalmol in press. 